\title{
USE OF MALDI-TOF MS IN DIAGNOSTIC MICROBIOLOGY FOR IDENTIFICATION OF SPECIES THAT CONVENTIONAL METHODS USUALLY FAIL TO IDENTIFY
}

\author{
Max Roberto Batista Araújo1, Luan Freitas Lana², \\ Luisa Ferreira Seabra ${ }^{1}$
}

\begin{abstract}
Matrix-assisted laser desorption/ionization time-of-flight mass spectrometry (MALDI-TOF MS) has been used in clinical diagnostic laboratories for the identification of microorganisms. It has a relevant advantage compared to other methods in terms of speed to provide results, being an alternative for addressing restrictions in clinical diagnosis as it may replace or complement existing identification techniques. This is especially important because some rare microorganisms would be identified only by higher cost techniques which are not widely available, such as genetic sequencing. Thus, the present paper reports two cases in which uncommon microorganisms were identified effectively and quickly.
\end{abstract}

Keywords: Non-tuberculous mycobacteria; Mycobacterium abscessus; bacterial infections; Actinotignum schaalii; spectrometry; mass, matrix-assisted laser desorption-ionization

Until recently, microbial identification in clinical diagnostic laboratories relied mainly on conventional phenotypic and gene sequencing identification techniques. The development of matrix-assisted laser desorption/ionization time-of-flight mass spectrometry (MALDI-TOF MS) devices has revolutionized the routine identification of microorganisms in clinical microbiology laboratories ${ }^{1}$. Thus, urinary tract infections (UTIs) and other infections caused by mycobacteria involving microorganisms that are uncommon in laboratory routine can be investigated.

The genus Mycobacterium consists of several species, including nontuberculous mycobacteria (NTM). The $M$. abscessus complex is a group of fast-growing, multiresistant and ubiquitous soil and water species that can cause infections more difficult to treat due to resistance to antimicrobial drugs. They are also resistant to the action of disinfectants and are responsible for post-surgery infections in almost all human organs ${ }^{2,3}$. Actinotignum schaalii is another rare microorganism that causes UTI and whose identification by traditional phenotypic testing is unusual, which may have obscured its role as a pathological agent for years.

Thus, considering the difficulties involved in the identification of these microorganisms, this paper reports a case of $M$. abscessus in peritoneal effluent culture and a case of $A$. schaalii in a patient with UTI.

\section{CASE REPORT}

A 61-year-old woman living in a municipality in Minas Gerais state, southeastern Brazil, presented with chronic renal failure undergoing periodic hemodialysis. She underwent peritoneal dialysis due to other acquired complications. She reported fever and abdominal pain; after collection, perversion of the peritoneal fluid was observed. Due to suspected infection, the catheter was withdrawn, the material was sent for analysis, and treatment was initiated with cefazolin and gentamicin. In the absence of clinical improvement, treatment was changed to vancomycin and ceftazidime, but her status remained unchanged.

http://seer.ufrgs.br/hcpa

(cc)BY

ISSN 2357-9730
Clin Biomed Res. 2019;39(2):171-174

1 Núcleo Técnico Operacional, Setor de Microbiologia, Instituto Hermes Pardini. Vespasiano, MG, Brasil.

2 Unidade de Apoio Operacional, Hospital das Clínicas, Universidade Federal de Minas Gerais (UFMG). Belo Horizonte, MG, Brasil.

Corresponding author: Max Roberto Batista Araújo max_barau@hotmail.com Instituto Hermes Pardini Av. das Nações, 2448. 33200-000, Vespasiano, MG, Brasil. 
The collected peritoneal effluent was plated in blood agar (BAP) (bioMérieux ${ }^{\circledR}$, Brazil), with bacterial growth occurring after 48 hours at $37^{\circ} \mathrm{C}$ (Figure 1). The colonies were assessed using the VITEK ${ }^{\circledR}$ 2 Compact (bioMérieux ${ }^{\circledR}$ ) automated system, but no microorganism was identified.

The colonies were then assessed using the VITEK ${ }^{\circledR}$ MS (bioMérieux ${ }^{\circledR}$ ) automated system, which identified them as $M$. abscessus (99\% reliability), as depicted by a characteristic protein fingerprint, not requiring the use of an extraction protocol. The identification was confirmed by polymerase chain reaction (PCR) followed by PCR-restriction fragment length polymorphism (PCR-RFLP), revealing the bands ${ }^{4}$. Based on the banding profile (Figure 2 ), the species was identified using an international database (http://app.chuv.ch/prasite/index.html). The primers used were TB11 (ACCAACGATGGTGTGTCCAT) and TB12 (CTTGTCGAACCGCATACCCT), with a coding sequence for a heat shock protein (HSP) recommended for identification of microorganisms of that genus. Genus and species were identified, but subspecies could not be determined with a sufficient degree of reliability.

In addition, there was a second unusual bacterial identification in our laboratory routine. A67-year-old man living in a municipality in Paraná state, southern Brazil, had undergone surgery to remove the prostate approximately six months earlier due to a malignant tumor. In the postoperative period, he used a urinary catheter, which was manipulated by himself or by family members.

In July 2018, the patient was diagnosed with UTI caused by extended-spectrum $\beta$-lactamase (ESBL)-producing Klebsiella pneumoniae at a count above $100,000 \mathrm{CFU} / \mathrm{mL}$. The treatment of choice was amikacin and imipenem, which led to clinical improvement and absence of pyuria. Therapeutic success was confirmed with a new urine culture showing a negative result.

Subsequently, in September 2018, symptoms of UTI reappeared. The clinician in charge opted for empiric treatment with sulfamethoxazole-trimethoprim, with no clinical response and maintenance of intermittent fever and pain during urination.

A urine sample was collected and plated onto blood agar and chromogenic agar media, showing no bacterial growth. Because of the patient's history, a Gram stain was performed, revealing unusual Gram-positive coccobacilli. The sample was then plated onto blood agar and, after 72 hours of anaerobic incubation, showed bacterial growth above $100,000 \mathrm{CFU} / \mathrm{mL}$.

Based on the comparison of the super spectrum obtained with reference spectra from the SARAMIS ${ }^{\circledR}$

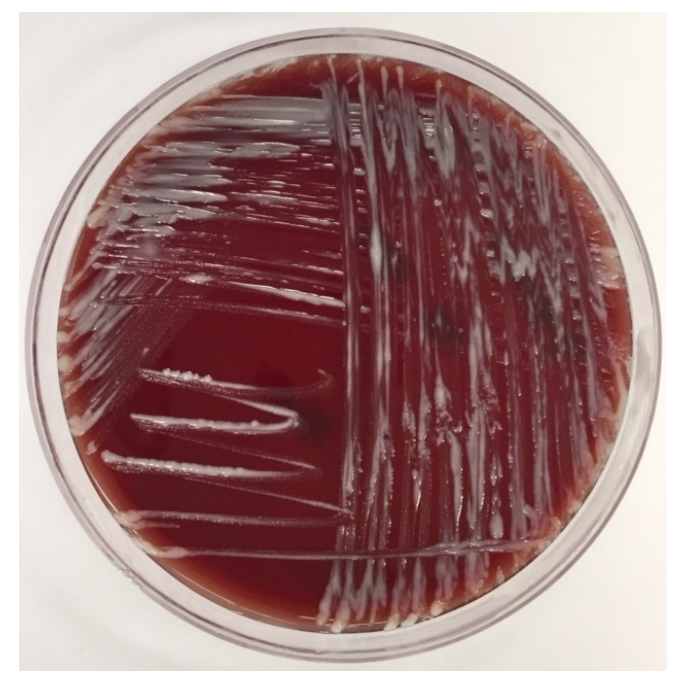

Figure 1: Colonies of $M$. abscessus on a blood agar plate.



Figure 2: Representative PCR-RFLP result amplified from the clinical isolate, showing the use of enzymes HAE (first three columns) and BSTE (last three columns). 


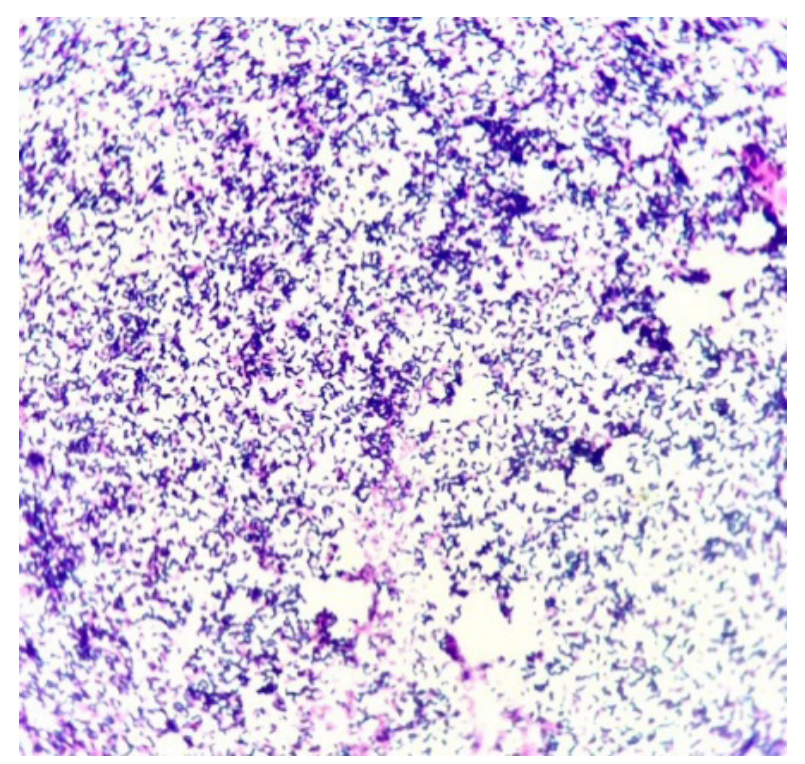

Figure 3: Gram staining (original magnification, ×1000) showing Gram-positive coccoid rods.

database (bioMérieux ${ }^{\circledR}$ ), A. schaalii was identified with $99.9 \%$ reliability. A Gram stain of the colonies was performed and Gram-positive coccobacillary forms were visualized by microscopy, which was consistent with the identification of the species (Figure 3).

In the absence of known standardization, antimicrobial susceptibility testing was not performed. Only the identification of the etiological agent was reported.

\section{DISCUSSION}

Among fast-growing mycobacteria, the M. abscessus complex has proved to be one of the most resistant to antibiotics, being involved in several types of community-acquired infections ${ }^{2}$. In this context, infections in patients on hemodialysis have been reported, with some progressing to widely disseminated diseases, demonstrating the virulence potential of those mycobacteria and their resistance to commonly used disinfectants, such as formaldehyde in concentrations below $2 \%$. This represents an increased risk of infections associated with health care ${ }^{5}$. After the identification of the microorganism, vancomycin and piperacillin-tazobactam were prescribed for 7 days, which, in contrast to previous treatments, had a good response and led to the improvement in the clinical status of the patient. Such therapy differs from what is traditionally indicated, but the response has been satisfactory. Moreover, there is a lack of consensus on optimal antimicrobial agents and combination therapy as well as optimal treatment duration and introduction of new antimicrobial agents (e.g., tigecycline) ${ }^{6}$.

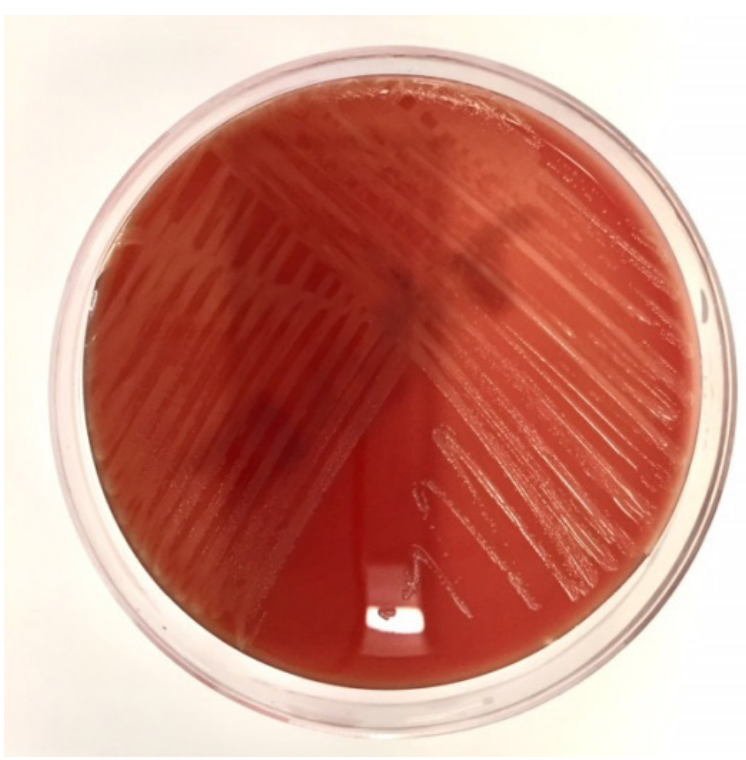

Figure 4: Colonial morphology of $A$. schaalii grown on a blood agar plate after 72 hours of incubation under anaerobic conditions at $37^{\circ} \mathrm{C}$.

Regarding UTI, clinical diagnostic laboratories usually incubate urine samples for 16 to 24 hours in aerobic conditions. This may be an impediment to $A$. schaalii isolation, as this is a facultative anaerobe that grows slowly (4 to 5 days) in blood agar medium enriched in $5 \%$ carbon dioxide or in anaerobic conditions ${ }^{7}$ (Figure 4). Studies have shown that this microorganism has infection rates that increase with age, being more common in patients over 60 years of age and with a history of $\mathrm{UTI}^{8}$, which is consistent with the characteristics of the patient of the present study.

Clinicians must consider the possibility of $A$. schaalii infection in patients predisposed to common or unexplained chronic UTI, especially if the microscopic result of the urine sample shows coccobacilli and leukocytes, with negative bacterial growth by aerobic culture and non-response treatment with ciprofloxacin or trimethoprim ${ }^{9}$. An erroneous diagnosis of Gardnerella vaginalis has also been reported in situations which the result was based only on Gram staining, colony appearance, and slow growth ${ }^{10}$.

With regard to treatment, two weeks of $\beta$-lactam is usually effective unless the patient has chronic prostatitis, in addition to 4 to 6 weeks of antibiotics that have the ability to cross the blood-prostate barrier ${ }^{11}$. Finally, for the present case report, the clinical status of the patient improved after therapy with imipenem was reinitiated.

\section{Conflicts of Interest}

The authors declare no conflicts of interest. 


\section{REFERENCES}

1. Croxatto A, Prod'hom G, Greub G. Applications of MALDI-TOF mass spectrometry in clinical diagnostic microbiology. FEMS Microbiol Rev. 2012;36(2):380-407. http://dx.doi.org/10.1111/j.15746976.2011.00298.x. PMid:22092265.

2. Brown-Elliott BA, Wallace RJ JR. Clinical and taxonomic status of pathogenic nonpigmented or late-pigmenting rapidly growing mycobacteria. Clin Microbiol Rev. 2002;15(4):716-46. http://dx.doi. org/10.1128/CMR.15.4.716-746.2002. PMid:12364376.

3. Griffith DE, Aksamit T, BrownElliott BA, Catanzaro A, Daley C, Gordin F, et al. An official ATS/IDSA statement: diagnosis, treatment, and prevention of nontuberculous mycobacterial diseases. Am J Respir Crit Care Med. 2007;175(4):367-416. http://dx.doi.org/10.1164/rccm.200604571ST. PMid:17277290.

4. Devallois A, Goh KS, Rastogi N. Rapid identification of mycobacteria to species level by PCR-restriction fragment length polymorphism analysis of the hsp65 gene and proposition of an algorithm to differentiate 34 mycobacterial species. J Clin Microbiol. 1997;35(11):2969-73. PMid:9350770.

5. Bolan G, Reingold AL, Carson LA, Silcox VA, Woodley CL, Hayes PS, et al. Infections with Mycobacterium chelonae in patients receiving dialysis and using processed hemodialyzers. J Infect Dis. 1985;152(5):1013-9. http:// dx.doi.org/10.1093/infdis/152.5.1013. PMid:4045242.

6. Sassi M, Drancourt M. Genome analysis reveals three genomospecies in Mycobacterium abscessus. BMC Genomics. 2014;15(1):359. http:// dx.doi.org/10.1186/1471-2164-15-359. PMid:24886480.

7. Olsen $A B$, Andersen PK, Bank S, Søby KM, Lund L, Prag J. Actinobaculum schaalii, a commensal of the urogenital area. BJU Int. 2013;112(3):394-7. http://dx.doi.org/10.1111/j.1464410X.2012.11739.x. PMid:23350855.
8. Reinhard M, Prag J, Kemp M, Andresen K, Klemmensen B, Hojlyng $\mathrm{N}$, et al. Ten cases of Actinobaculum schaalii infection: clinical relevance, bacterial identification, and antibiotic susceptibility. J Clin Microbiol. 2005;43(10):5305-8. http://dx.doi. org/10.1128/JCM.43.10.53055308.2005. PMid:16208004.

9. Bank S, Jensen A, Hansen TM, Søby KM, Prag J. Actinobaculum schaalii, a common uropathogen in elderly patients, Denmark. Emerg Infect Dis. 2010;16(1):76-80. http:// dx.doi.org/10.3201/eid1601.090761. PMid:20031046.

10. Horton LE, Mehta SR, Aganovic L, Fierer J. Actinotignum schaalii infection: a clandestine cause of sterile pyuria?. Open Forum Infect Dis. 2018;5(2):ofy015. PMid: 29450211. http://doi.org/ 10.1093/ofid/ofy015.

11. Meares EM JR. Prostatitis: review of pharmacokinetics and therapy. Rev Infect Dis. 1982;4(2):475-83. http:// dx.doi.org/10.1093/clinids/4.2.475. PMid:7051242. 